\title{
The case for too little melatonin signalling in increased diabetes risk
}

\author{
Amélie Bonnefond $^{1,2} \cdot$ Philippe Froguel ${ }^{1,2}$
}

Received: 13 December 2016/Accepted: 6 March 2017 /Published online: 17 March 2017

(C) Springer-Verlag Berlin Heidelberg 2017

\begin{abstract}
Genome-wide association studies have detected an association between type 2 diabetes risk and a non-coding SNP located in MTNR1B, the gene encoding melatonin receptor 2 (MT2). Melatonin regulates circadian rhythms and sleep and associates with metabolic disorders. However, the mechanisms underlying these actions are still unclear. Functional genomic, animal and clinical studies have not reached the same conclusions: while some studies have reported that decreased melatonin signalling increases type 2 diabetes risk, others have found the opposite. In this commentary, we have tried to provide an explanation for these contradictions and we suggest how the community may progress to reach a unified picture of the effect of melatonin and its signalling on type 2 diabetes.
\end{abstract}

Keywords eQTL · Genome-wide association study · GWAS · Melatonin $\cdot$ Melatonin receptor $\cdot M T N R 1 B / M T 2 \cdot$ Pancreatic islets $\cdot$ Rare variants $\cdot$ Sequencing $\cdot$ SNP

\section{Abbreviations \\ GWAS Genome-wide association studies \\ LoF Loss-of-function}

Amélie Bonnefond

amelie.bonnefond@inserm.fr

Philippe Froguel

pfroguel@ic.ac.uk

1 European Genomic Institute for Diabetes (EGID), Institut Pasteur de Lille, Centre national de la recherche scientifique (CNRS), Unité mixte de recherche (UMR) 8199, University of Lille, 1 Rue du Professeur Calmette, B.P. 245, F-59019 Lille Cedex, France

2 Department of Genomics of Common Disease, School of Public Health, Hammersmith Hospital, Imperial College London, Du Cane Road, London W12 0NN, UK
MT2 Melatonin receptor 2

NGS Next-generation sequencing

QTL Quantitative trait loci

eQTL Expressed transcript quantitative trait loci

\section{Introduction}

A strong debate has recently arisen regarding the effect of melatonin on type 2 diabetes risk. Following a publication stating that enhanced melatonin signalling increases the risk of type 2 diabetes [1], two commentaries had opposite views on the relevance of these data $[2,3]$. This question is crucial as melatonin is available over-the-counter as a dietary supplement for its anti-oxidant/anti-ageing effect, and/or to treat acute or chronic sleep problems. In the USA, there was a significant increase in the use of melatonin: from $0.1 \%(n=87,000)$ in 2007 to $0.7 \%$ $(n=419,000)$ in 2012, among children; and from $0.6 \%$ $(n=1,296,000)$ in 2007 to $1.3 \%(n=3,065,000)$ in 2012, among adults $[4,5]$. Therefore its safety is of major importance.

\section{The variable effect of melatonin}

Melatonin is one of the most ubiquitous and versatile molecules [6]. Its presence has been shown in bacteria, unicellular eukaryotes, algae, plants, fungi, and in invertebrates and vertebrates, which suggests a high evolutionary age [6]. High levels of melatonin were reported in Chinese medicinal herbs traditionally used for delaying age-related disorders [6]. Thus, at first sight, melatonin seems to be physiologically produced and not harmful to life.

Melatonin is primarily secreted from the pineal gland throughout the night in both diurnal and nocturnal vertebrates, where it is the major timekeeping hormone. Importantly, 
depending on the species, melatonin can exert opposing effects on specific functions. For instance, melatonin directly stimulates vocalisation in a nocturnally breeding 'singing' teleost fish, while it inhibits vocal activity in diurnal birds during the night [7]. Therefore, in studies that aim to analyse the effect of melatonin physiologically, it is crucial to be aware that results found in nocturnal rodents can be opposite to those in diurnal humans. For instance, there are conflicting in vitro studies on the impact of melatonin on insulin secretion from rodent and human islets; in rodent beta cells melatonin was shown to decrease insulin secretion $[1,8]$, whilst in one study of human islets, melatonin was observed to potentiate insulin secretion [9].

\section{Melatonin signalling via its receptors}

Melatonin has two cell-membrane receptors in mammals: melatonin receptor 1 (MT1; encoded by MTNR1A) and melatonin receptor 2 (MT2; encoded by MTNR1B), which are inhibitory G-protein coupled $\left(\mathrm{G}_{\mathrm{i}}\right.$-coupled) receptors. However, as melatonin is a small lipophilic molecule, it has very high cell membrane permeability [10]. Therefore, melatonin can cross cell membranes alone, without the involvement of its receptors, making studies on the physiology of melatonin challenging and possibly misleading. In this respect, the inhibitory effects of melatonin on insulin secretion from rodent beta cells could be independent of melatonin receptors.

In this context, through genome-wide association studies (GWAS) we and others have found that the minor allele of the common DNA variant (rs10830963) in the only intron of $M T N R 1 B$ is associated with increased type 2 diabetes risk and with variation of several related metabolic traits in humans [11]. This MT2-associated discovery was attractive as the relationship between circadian rhythms, glucose homeostasis and related diseases had already been shown in multiple reports.

\section{Decreased melatonin signalling and type 2 diabetes risk}

Subsequent to these GWAS, our group performed a large-scale resequencing of the coding exons of MTNR1B in almost 8000 samples. This was followed by a four-tier functional investigation of each mutant using the human embryonic kidney (HEK) cell line. We found that only loss-of-function (LoF) MTNR1B variants, and not benign variants, associate with increased type 2 diabetes risk in response to melatonin [12]. This work was based on a comprehensive assessment of the effects of the mutations on several signalling pathways (including but not exclusive to cAMP) or functions triggered by melatonin via its receptor. Our data suggested that decreased melatonin signalling through deleterious MT2 receptor activity increases type 2 diabetes risk. These conclusions were in line with various studies done in rodents, beta cells and human cohorts, which showed that high melatonin levels, or the administration of melatonin or of melatonergic agonists, lead to decreased (and not augmented) risk of diabetes [9, 13, 14]. Indeed, for instance, a 12 year prospective study including 370 women who developed type 2 diabetes and 370 matched control participants showed that decreased levels of melatonin secretion during the night is independently associated with a higher risk of developing type 2 diabetes [13]. However, Tuomi et al showed in 45 individuals that 3 month exposure to a high dose of melatonin (4 mg per day) is associated with higher glucose values $90 \mathrm{~min}$ post glucose load [1]. Thus, the benefit of future studies on this specific topic is essential, so as to reconcile these discrepancies.

To date, there is unfortunately no independent comprehensive replication of our genetic and functional study, although an unpublished US cohort study that included 6899 participants presented an association between a mutation (already detected in our study [12]) and increased type 2 diabetes risk at a recent meeting [15]. Moreover, in a recent huge nextgeneration sequencing (NGS) study the GoT2D and T2DGENES consortia failed to detect the total LoF mutations that are associated with type 2 diabetes risk (including the total LoF p.L60R mutation, which has a frequency of $0.06 \%$ in the European population according to the National Heart, Lung, and Blood Institute Grand Opportunity [NHLBI GO] Exome Sequencing Project and the Exome Aggregation Consortium), possibly because of the low read depth at the MTNR1B first exon through NGS [16]. Therefore, further studies are clearly needed to confirm or invalidate our study.

On the other hand, several studies have reported that the type 2 diabetes risk allele rs10830963 is associated with increased MTNR1B mRNA level in human pancreatic islets [1, 2], considered to imply that increased type 2 diabetes risk can be due to triggered melatonin signalling. However, increased RNA content does not necessarily imply enhanced abundance of the related protein. Indeed, using genetic, transcriptome and proteome data, two independent groups have reported that the overlap between expressed transcript quantitative trait loci (eQTL) and protein quantitative trait loci (QTL) is low in both humans and mice: less than $30 \%$ of eQTL were translated into protein QTL in both studies $[17,18]$.

\section{MTNR1B expression in human islets}

In humans, the true presence of MT2 protein in islets is actually uncertain; MT2 protein expression has never been reported in human islets (in contrast to different parts of the brain, where it is abundant) following western blot analysis, despite huge efforts from the research community. Furthermore, according to recent single-cell transcriptome profiling of human 
pancreatic islets, MTNR1B transcripts are detectable in less than $5 \%$ of human islet single cells [19]. This is in line with more conventional RNA sequencing data showing that $M T N R 1 B$ expression is extremely low in the human pancreatic beta cell line EndoC- $\beta \mathrm{H} 1$ and in primary beta cells, with a reads per kilobase of transcript per million mapped reads (RPKM) score of 0.015 and 0.64 , respectively [20]. These data suggest it is unlikely that the MTNR1B eQTL are highly significant in terms of whole-body (patho)physiology through direct beta cell impairment, although the scientific community should continue its effort in the analysis of MTNR1B/MT2 expression in pancreatic islets. The investigation of beta cellspecific Mtnrlb deletion in mice may also reveal some clues about the role of MT2 in pancreatic islets, although mouse models are not optimal for the investigation of melatonin physiology.

\section{Conclusions}

In conclusion, the investigation of the role of melatonin signalling in insulin secretion is difficult in humans and the mechanism by which the type 2 diabetes-associated MTNRIB rs10830963 SNP impairs whole-body insulin secretion is still a mystery. Since melatonin is primarily a master regulator of the circadian clock, we believe that both MTNR1B rs10830963 and $M T N R 1 B$ rare LoF variants increase type 2 diabetes risk via an impairment in the central function of melatonin on circadian rhythms. In line with this hypothesis, a recent study has shown that MTNR1B rs10830963 has a significant effect on the dynamics of melatonin secretion [21]. We propose that rs10830963 may affect the transcription of several genes (including MTNR1B) in isolated regions of the brain (such as the pineal glands that secrete melatonin); defects in insulin secretion may be a secondary effect of central dysfunction. Future studies (circadian/sleep phenotyping) using carriers of $M T N R 1 B$ rare LoF mutations and carriers of the MTNR1B risk allele rs 10830963 are warranted to confirm this hypothesis.

Funding Work related to melatonin in the authors' laboratory is supported by the EASD/Janssen ('Rising Star', awarded to AB) and by the Agence Nationale de la Recherche (ANR-2011-BSV1-012-01 'MLT2D' and ANR-2011-META "MELA-BETES", awarded to AB and PF). $A B$ is supported by Inserm.

Duality of interest The authors declare that there is no duality of interest associated with this manuscript.

Contribution statement $\mathrm{AB}$ and $\mathrm{PF}$ wrote the article and revised it critically for important intellectual content. $\mathrm{AB}$ and $\mathrm{PF}$ approved the version to be published.

\section{References}

1. Tuomi T, Nagorny CLF, Singh P et al (2016) Increased melatonin signaling is a risk factor for type 2 diabetes. Cell Metab 23:1067-1077

2. Persaud SJ, Jones PM (2016) A wake-up call for type 2 diabetes? N Engl J Med 375:1090-1092

3. Bonnefond A, Karamitri A, Jockers R, Froguel P (2016) The difficult journey from genome-wide association studies to pathophysiology: the melatonin receptor 1B (MT2) paradigm. Cell Metab 24: 345-347

4. Clarke T, Black L, Stussman B, et al (2015a) Use of complementary health approaches among children aged 4-17 years in the United States: National Health Interview Survey, 2007-2012. Natl Health Stat Report 78

5. Clarke T, Black L, Stussman B, et al (2015b) Trends in the use of complementary health approaches among adults: United States, 2002-2012. Natl Health Stat Report 79

6. Pandi-Perumal SR, Srinivasan V, Maestroni GJM et al (2006) Melatonin: nature's most versatile biological signal? FEBS J 273: 2813-2838

7. Feng NY, Bass AH (2016) "Singing" fish rely on circadian rhythm and melatonin for the timing of nocturnal courtship vocalization. Curr Biol 26:2681-2689

8. Lyssenko V, Nagorny CLF, Erdos MR (2009) Common variant in MTNR1B associated with increased risk of type 2 diabetes and impaired early insulin secretion. Nat Genet 41:82-88

9. Costes S, Boss M, Thomas AP, Matveyenko AV (2015) Activation of melatonin signaling promotes $\beta$-cell survival and function. Mol Endocrinol 29:682-692

10. Yu H, Dickson EJ, Jung S-R et al (2016) High membrane permeability for melatonin. J Gen Physiol 147:63-76

11. Bonnefond A, Lyssenko V (2016) From association to function: MTNR1B. In: Florez JC (ed) The genetics of type 2 diabetes and related traits. Springer International Publishing, Cham, Switzerland, pp 403-421

12. Bonnefond A, Clément N, Fawcett K et al (2012) Rare MTNR1B variants impairing melatonin receptor $1 \mathrm{~B}$ function contribute to type 2 diabetes. Nat Genet 44:297-301

13. McMullan CJ, Schernhammer ES, Rimm EB et al (2013) Melatonin secretion and the incidence of type 2 diabetes. JAMA 309:1388-1396

14. Hardeland R (2017) Melatonin and the pathologies of weakened or dysregulated circadian oscillators. J Pineal Res 62:e12377

15. Bansal V, Gassenhuber J, Phillips T, et al (2014) Targeted sequencing of genes associated with type 2 diabetes in 6800 individuals. Am Soc Hum Genet - Meet Abstr 760S

16. Fuchsberger C, Flannick J, Teslovich TM et al (2016) The genetic architecture of type 2 diabetes. Nature 536:41-47

17. Williams EG, Wu Y, Jha P et al (2016) Systems proteomics of liver mitochondria function. Science 352:aad0189

18. Battle A, Khan Z, Wang SH et al (2015) Impact of regulatory variation from RNA to protein. Science 347:664-667

19. Segerstolpe $\AA$, Palasantza A, Eliasson P et al (2016) Single-cell transcriptome profiling of human pancreatic islets in health and type 2 diabetes. Cell Metab 24:593-607

20. Thomsen SK, Ceroni A, van de Bunt M et al (2016) Systematic functional characterization of candidate causal genes for type 2 diabetes risk variants. Diabetes 65:3805-3811

21. Lane JM, Chang A-M, Bjonnes AC et al (2016) Impact of common diabetes risk variant in MTNR1B on sleep, circadian, and melatonin physiology. Diabetes 65:1741-1751 